Pak. j. sci. ind. res. Ser. A: phys. sci. 2017 60(2) 85-89

\title{
A Three-Layer Microstrip Resonator for Complex Permittivity Measurement of Medium Loss Liquids Using 3D-FDTD Simulation
}

\author{
Cyrus Avaznia ${ }^{a}$ and Gholamreza Moradi ${ }^{\text {bc* }}$ \\ ${ }^{a}$ Department of Electrical Engineering, Amirkabir University of Technology, Tehran-15914, Iran \\ ${ }^{b}$ Wave Propagation and Microwave Measurement Research Laboratory, Department of Electrical Engineering, \\ Amirkabir University of Technology, Tehran-15914, Iran \\ ${ }^{c}$ Mechanical and Electrical Engineering Departments, University of Alberta, Edmonton, Canada
}

(received October 15, 2016; revised April 8, 2017; accepted April 27, 2017)

\begin{abstract}
A three-layer microstrip resonator is introduced to measure complex permittivities of medium loss liquids. The device is configured such that the first layer containing the liquid under test; a sheet of polytetrafluoroethylene (PTFE) is embedded in the middle layer, and the third layer functions as the base on which the patch is printed. The base layer is inverted on PTFE layer, and reflection coefficient is measured from $2.5 \mathrm{GHz}$ to $5 \mathrm{GHz}$. The complex permittivities are extracted from the resonant frequency and the 10-dB bandwidth of S-parameter for different combinations of ethanol and methanol. Indeed, a three-layer microstrip resonator allows us to possess an affordable, and yet, high-accuracy electrical device to measure complex permittivities of medium loss liquids. FDTD method is used for analysing the structure and the results obtained by using FDTD method and the experimental data indicate a high degree of similarity.
\end{abstract}

Keywords: microstrip resonator, microwave chemistry, complex permittivity measurement, 3D-FDTD

\section{Introduction}

Complex permittivity measurement is of great significance in bio-electromagnetics, microwave chemistry and microwave engineering. Complex permittivity of some biological dilutions, which is important for millimeter-wave dosimetry studies, has been extracted by means of an open-ended coaxial probe (Zhadobov et al., 2012). A microwave resonator has been constructed to characterize the complex permittivity of fruitful solutions in chemistry and biology (Chretiennot et al., 2013) and several methods have been recently investigated to measure the dielectric of a ferroelectric composition, which is used in the electronics industry (Queffelec et al., 2014). The permittivity of many substances changes with frequency and quantifying of the permittivity of these materials with high resolution along with frequency, specifically microwave frequencies, is of prime concern in different industries (Sarri et al., 2012; Osman et al., 2008, Komarov et al., 2005). Planar circuits have always been one of the best instruments to determine the complex permittivity of materials (Chakyar et al., 2017, Yang et al., 2016; Ansari et al., 2015). They have great advantages including small size, light weight, low power consumption, and easy imple-

*Author for correspondence; E-mail: ghmoradi@aut.ac.ir mentation. On the other hand, the number of methods applicable to measure their permittivities is reduced by the lack of particular shape in liquids. Microstrip resonators, as one type of planar circuits, have shown a good performance in the permittivity measurement of liquids. Given the importance of identification of liquids in applied sciences, resonant methods make a considerable contribution; through exploring the complex permittivity of liquids with an eligible degree of accuracy and sensitivity (Yu et al., 2000).

A three-layer microstrip resonator is introduced, by which the complex permittivity of liquids is determined. The resonator is composed of three substrates; the first substrate is considered to measure the sample liquid, the second substrate is fixed by a Teflon (PTFE) sheet which is used to increase the degree of freedom in choosing resonant frequency, and the last is the base layer on which the patch is inversely fabricated. The resonator measures the complex permittivity of medium loss liquids for the frequency near and below $4 \mathrm{GHz}$.

\section{Materials and Methods}

Numerical analysis. Finite-difference time-domain (FDTD) was initially proposed in 1966 by Yee (1966). This modeling technique, which solves Maxwell's 
equations in time domain, has been broadly used to simulate various electromagnetics problems (Sullivan, 2013; Taflone and Susan, 2000). FDTD method is very appropriate to model dispersive material in a wide range of frequencies (Bia et al., 2015; Luebbers et al., 1990, Yee, 1966). The $S_{11}$ parameter of binary mixtures of ethanol-methanol, which belong to dispersive materials characterised by Debye Law (Bao et al., 1996) was simulated by using this time domain method.

A three dimensional FDTD code is written for the resonator. The code is implemented by MATLAB. This code has been used to validate and compare visually the results of the measurements with standard values.

Extraction of complex permittivity components. Notwithstanding, there are some equations that represent resonant frequency and Q-factor are dependent on the real $\left(\epsilon^{\prime}\right)$ and imaginary $\left(\epsilon^{\prime \prime}\right)$ parts of the complex permittivity of the substrates (Gupta and Srivastava, 2012). To extract the complex permittivity components, an approximate approach was considered which is simpler and more practical than the mentioned complex equations (Gupta and Srivastava, 2012). There is almost an inverse relationship between the resonant frequency $f_{0}$ and the magnitude of $\in^{\prime}$. In the same way, an inverse relationship can be considered between Q-factor and the quantity of $\in$ ". Equations (1) and (2) illustrate these issues. The results of simulations and experiments presented confirm these assumptions. In the FDTD code, the values of $\epsilon$ ' and $\in$ " are dependent on frequency and are extracted from Debye function (Bao et al., 1996).

$$
\begin{aligned}
& \frac{\epsilon_{1}^{\prime}}{\epsilon^{\prime}{ }_{2}} \approx \frac{f_{2}}{f_{1}} . \\
& \frac{\epsilon^{\prime \prime}}{\epsilon^{\prime \prime}}=\frac{\mathrm{Q}_{2}}{\mathrm{Q}_{1}} .
\end{aligned}
$$

As reported by Bao et al. (1996), the changes of the real and imaginary part of the complex permittivity of ethanol-methanol binary mixtures along the intended frequency range are almost linear in connection with changes in volume fractions of these two liquids. The results are classified in the format of table and any unknown binary mixture of ethanol and methanol can be interpolated linearly from this table. Thus, the values of the real $\left(\epsilon_{\mathrm{x}}^{\prime}\right)$ and imaginary $\left(\epsilon_{\mathrm{x}}^{\prime \prime}\right)$ part of the complex permittivity can be extracted as:

$$
\frac{\epsilon_{\mathrm{x}-}^{\prime} \epsilon_{1}^{\prime}}{\epsilon_{2}^{\prime}-\epsilon_{1}^{\prime}}=\frac{f_{1}-f_{\mathrm{x}}}{f_{1-}-f_{2}}
$$

$$
\frac{\epsilon^{\prime \prime}-\epsilon_{1}^{\prime \prime}}{\epsilon_{2}^{\prime \prime}-\epsilon_{1}^{\prime \prime}}=\frac{\mathrm{BW}_{\mathrm{x}}-\mathrm{BW}_{1}}{\mathrm{BW}_{2}-\mathrm{BW}}
$$

Where:

constants of $\epsilon_{1}^{\prime}, \in^{\prime \prime}, \in_{2}^{\prime}$ and $\in^{\prime \prime}{ }_{2}$ are real, reference data by $\mathrm{Bao}$ et al. (1966). $f_{1}, f_{2}, \mathrm{BW}_{1}$ and $\mathrm{BW}_{2}$ are achieved by the FDTD code and $f_{\mathrm{x}}$ and $\mathrm{BW}_{\mathrm{x}}$ are obtained from the experimental results.

Minimization of errors of permittivity components. Like many electrical structures, there are some errors and discrepancies between measurement values delivered by device under test and standard ones. One of the most common and not to mention easiest way to minimize these errors is equally shifting primary results to the standard values for each component of the permittivity. But this kind of gradation cannot minimize all the errors since the real part and imaginary part of the complex permittivity in ethanol-methanol binary mixtures change nonlinearly with frequency. Nevertheless, a certain frequency zone may be designated in which both parts of the permittivity approximately vary linearly in the frequency domain. The frequency range from $3.5 \mathrm{GHz}$ to $4 \mathrm{GHz}$ would be an overall proper choice. Therefore, discrepancies between measured and standard values decreased conspicuously. Actually, this was attained because the three-layer microstrip resonator allowed to choose an appropriate frequency domain.

Three-layer microstrip resonator configuration. Among many design challenges, the expenditure in manufacturing a device has constantly been of prime concern. Apart from cost issue, availability of the material, having appropriate features; is a key factor. In many cases, the material, which is appropriate to realize a high-accuracy measuring device, is out of reach and providing the material would not be affordable. Achieving both of these is a difficult task. The threelayer microstrip resonator, however, seems to be able to fulfill this target. The presence of two substrates beside liquid under test enhances the number of materials that can be selected as substrates in association with our demands.

Schematic diagram of the proposed microstrip resonator configuration is shown in Fig. 1. The thickness of sample layer is $h_{\text {mut }}=1 \mathrm{~mm}$. PTFE is set in middle layer with thickness of $h_{t f l n}=2 \mathrm{~mm}$. The base layer is a polyester (RO4003) with relative permittivity $\epsilon_{\mathrm{r}}=3.4$ and thickness of $h_{\text {base }}=0.5 \mathrm{~mm}$. The cupric microstrip patch was designed in $28 \times 20 \mathrm{~mm}^{2}$ dimensions. The $2 \times 10 \mathrm{~mm}^{2}$ 
quarter-wavelength matched the $4 \times 10 \mathrm{~mm}^{2}$ microstrip transmission line to the patch. Also, SMA connector with flange jack was employed to supply the resonator. All layers were covered with a Teflon-metal enclosure; the metal portion under the structure had the duty of ground plane. Teflon layer above the resonator prevents the device from being aesthetically unpleasant. Both were jointly considered as an enclosure to protect substrates. Total dimensions of the system, as shown in Fig. 1, are $80 \times 70 \times 20 \mathrm{~mm}^{3}$.

\section{Results and Discussion}

The permittivity of different binary mixtures of ethanol and methanol was constructed by the resonator. The sample layer of the structure was filled by the binary mixtures, and then reflection coefficient was measured by a vector network analyzer in the frequency range of $2.5 \mathrm{GHz}$ to $5 \mathrm{GHz}$ (Fig. 2). This test was performed for eleven samples in which the volume fractions of ethanol and methanol varied in a scale of $10 \%$ - from $0 \%$ to $100 \%$. The results of the parameters were saved and then replotted in addition to FDTD results using MATLAB

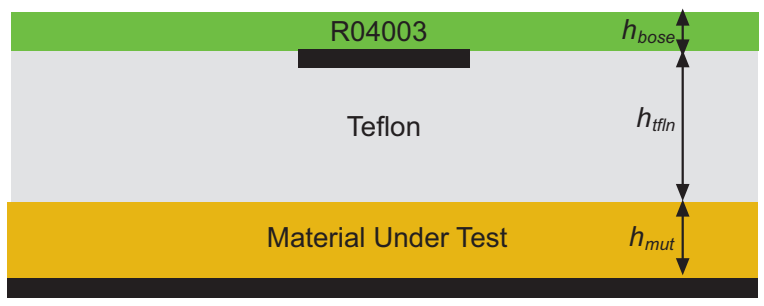

(a)

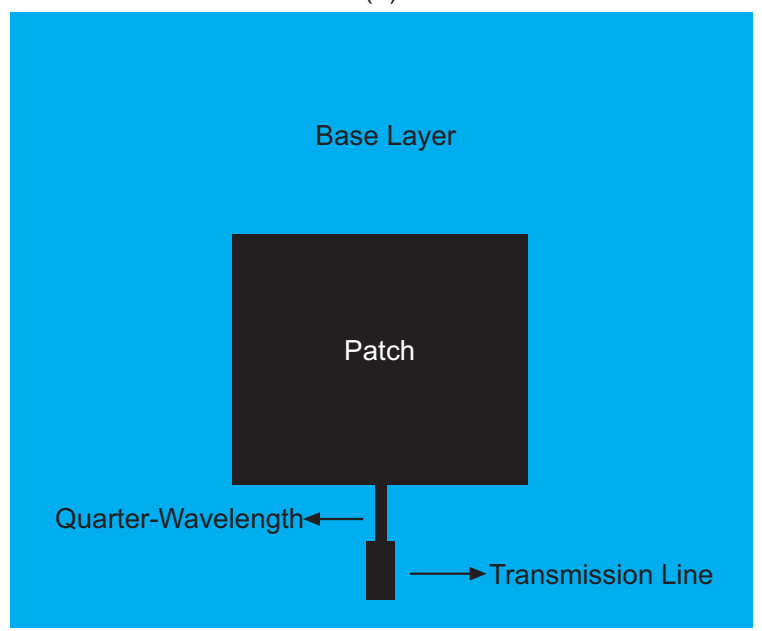

(b)

Fig. 1. Configuration of the three-layer microstrip resonator from (a) lateral view and (b) top view. in order to display visually the similarity between two approaches. Figure 3 shows two specimens of these plots. Due to good assumptions in FDTD code, the simulation speed of this numerical technique would be considerably less than simulator softwares, which can be investigated later. However, the main purpose of providing this numerical method has been offering an available approach to analyze our experimental results and data in Tables 1-2 demonstrate the success of this attempt.

As mentioned before, the complete results including amounts of measured and simulated resonant frequency and 10-dB bandwidth of all samples and errors of $\in$ ' and $\in$ " have been provided in Tables 1-2. The errors have been extracted according to equations (3) and (4) and also have been accomplished by minimization method, explained in second section (Minimization of
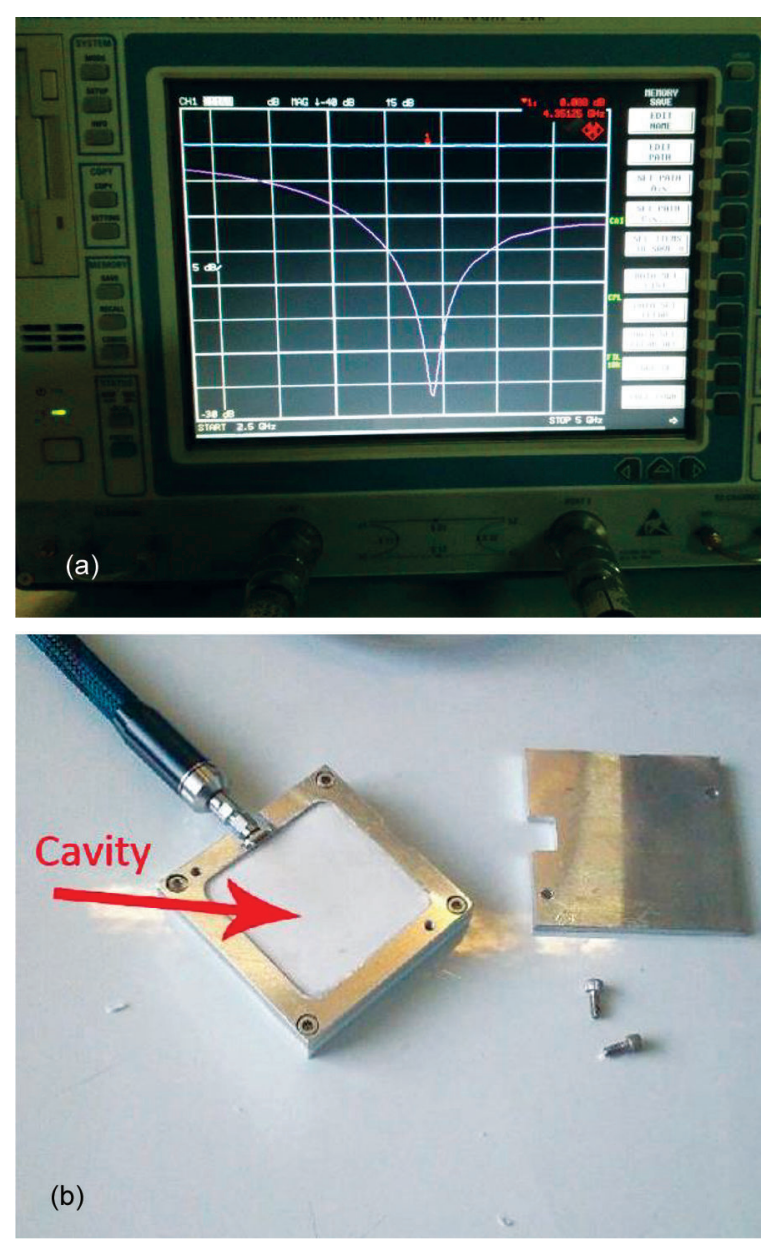

Fig. 2. Photo of (a) the measurement system and (b) the microstrip resonator. 
errors of permittivity components). The considerable achievement obtained by the new resonator was regarding the errors of the imaginary $\left(\epsilon^{\prime \prime}\right)$ part of the complex permittivity in the binary mixtures which keep limited. The resemblance between the $10-\mathrm{dB}$ bandwidths of the two curves in Fig. 3 demonstrates this claim. Capability in choosing a proper resonant frequency, which varied between $3.5 \mathrm{GHz}$ and $4 \mathrm{GHz}$, in a three-layer microstrip resonator led to acquire such an improvement. In this frequency area, both components of the complex permittivity showed almost linear changes with respect to changes in volume fractions of ethanol-methanol. In future works, the effect of feed system and matching can also be considered on the accuracy of measurements in the three-layer microstrip resonator by applying different types of feeding techniques.

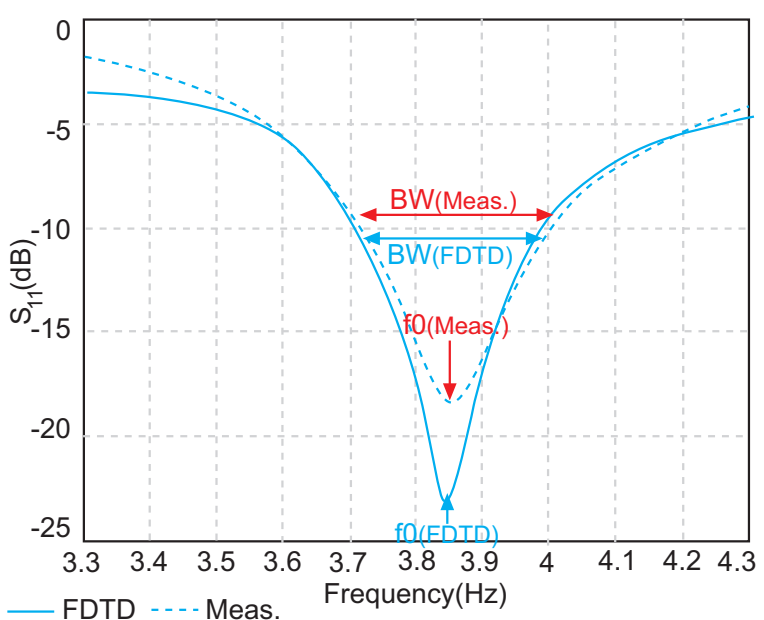

Fig. 3. Measured and simulated curves of reflection coefficient for binary mixture of ethanol $80 \%$ - methanol $20 \%$.

In Tables 1-2 the errors of the real and imaginary part of the complex permittivity have been given as:

$$
\text { Error }_{\epsilon} \mathrm{x} \quad(\%)=\frac{\epsilon_{\text {data }}^{\mathrm{x}}-\in^{\mathrm{x}}{ }_{\text {meas }}}{\epsilon_{\text {data }}^{\mathrm{x}}} \times 100
$$

Where:

$\epsilon_{\text {data }}^{\mathrm{x}}$ is the permittivity component retrieved from (Bao et al., 1996), and $\in^{\mathrm{x}}{ }_{\text {meas }}$ is obtained from measured results and equations (3) and (4).

In order to indicate improvement in measuring the complex permittivity of the binary mixtures of ethanol and methanol, the measured results by the three-layer microstrip resonator has been compared to the result of
Table 1. Results of measured and simulated resonant frequency of samples and measurement errors of $\left(\epsilon^{\prime}\right)$

\begin{tabular}{llll}
\hline \hline Material under test & $\begin{array}{l}\mathrm{f}_{0}(\mathrm{GHz})- \\
\text { FDTD }\end{array}$ & $\begin{array}{l}\mathrm{f}_{0}(\mathrm{GHz})- \\
\text { Measurement }\end{array}$ & $\begin{array}{l}\text { Error } \\
\left(\in{ }^{\prime}\right)\end{array}$ \\
\hline Ethanol 1.0 - Methanol 0.0 & 3.886 & 3.907 & $0.7 \%$ \\
Ethanol 0.9 - Methanol 0.1 & 3.868 & 3.889 & $1.2 \%$ \\
Ethanol 0.8 - Methanol 0.2 & 3.851 & 3.873 & $0.8 \%$ \\
Ethanol 0.7 - Methanol 0.3 & 3.838 & 3.859 & $2.9 \%$ \\
Ethanol 0.6 - Methanol 0.4 & 3.830 & 3.854 & $-1.2 \%$ \\
Ethanol 0.5 - Methanol 0.5 & 3.819 & 3.843 & $-1.5 \%$ \\
Ethanol 0.4 - Methanol 0.6 & 3.811 & 3.835 & $-1.2 \%$ \\
Ethanol 0.3 - Methanol 0.7 & 3.804 & 3.828 & $-2.8 \%$ \\
Ethanol 0.2 - Methanol 0.8 & 3.800 & 3.824 & $-2 \%$ \\
Ethanol 0.1 - Methanol 0.9 & 3.795 & 3.819 & $-1.8 \%$ \\
Ethanol 0.0 - Methanol 1.0 & 3.789 & 3.813 & $-1.6 \%$ \\
\hline \hline
\end{tabular}

Table 2. Results of measured and simulated $10-\mathrm{dB}$ bandwidth of samples and measurement errors of $\left(\epsilon^{\prime \prime}\right)$

\begin{tabular}{llll}
\hline \hline Material under test & $\begin{array}{l}\text { BW(MHz)- } \\
\text { FDTD }\end{array}$ & $\begin{array}{l}\text { BW(MHz)- } \\
\text { Measurement }\end{array}$ & $\begin{array}{l}\text { Error } \\
\left(\in{ }^{\prime \prime}\right)\end{array}$ \\
\hline Ethanol1.0 -Methanol0.0 & 426 & 427.3 & $2.8 \%$ \\
Ethanol0.9-Methanol 0.1 & 419 & 420.1 & $1.6 \%$ \\
Ethanol0.8-Methanol 0.2 & 405.1 & 406.9 & $0.7 \%$ \\
Ethanol0.7-Methanol 0.3 & 392.9 & 395.1 & $0.3 \%$ \\
Ethanol0.6-Methanol 0.4 & 380.4 & 382.5 & $0.8 \%$ \\
Ethanol0.5-Methanol 0.5 & 364.1 & 367.6 & $-1.1 \%$ \\
Ethanol0.4-Methanol 0.6 & 353 & 358.5 & $-3.2 \%$ \\
Ethanol0.3-Methanol 0.7 & 346 & 351 & $-1.8 \%$ \\
Ethanol0.2-Methanol 0.8 & 336 & 342 & $-4.1 \%$ \\
Ethanol0.1-Methanol 0.9 & 331 & 334.5 & $-0.7 \%$ \\
Ethanol0.0-Methanol 1.0 & 322.9 & 326.8 & $-0.9 \%$ \\
\hline \hline
\end{tabular}

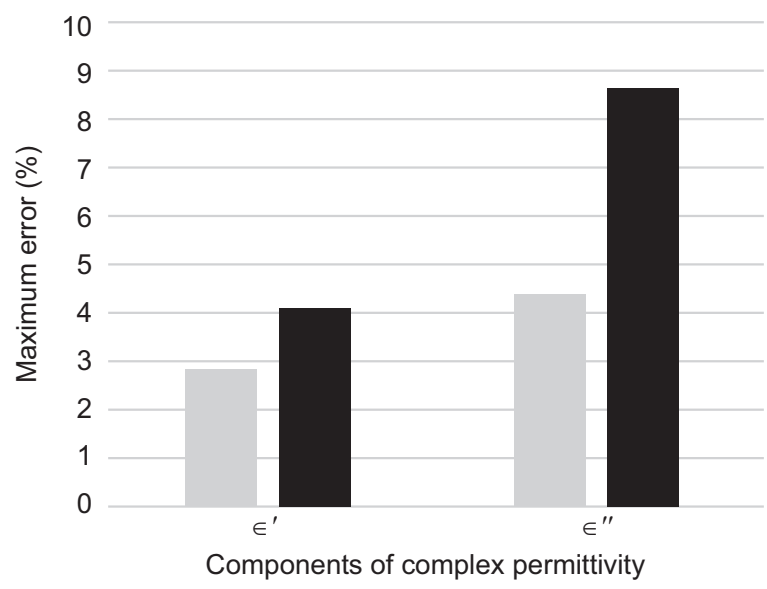

Three-Layer microstrip resonator

Microstrip resonator with slotted ground plane

Fig. 4. Maximum measurement errors for the real and imaginary part of the complex permittivity in the three-layer microstrip resonator and a microstrip resonator with slotted ground plane. 
a microstrip resonator with slotted ground plane (Liu and $\mathrm{Pu}, 2008$ ), and the maximum errors of the real and imaginary part of the complex permittivity measured by both instruments have been plotted in Fig. 4 .

\section{Conclusion}

A three-layer microstrip resonator was demonstrated in order to measure the complex permittivity of binary mixtures of ethanol-methanol. FDTD simulations and measurements were applied to illustrate the accuracy of the structure. The maximum measurement errors for the real and imaginary part of the complex permittivity were produced $2.9 \%$ and $4.1 \%$, respectively, indicating the high quality of this resonator. In fact, the presence of this configuration allowed us to choose its operating frequency and materials used in the resonator. The former increased the precision of measurements; the later decreased the expenditure of the structure.

\section{References}

Ansari, M.A.H., Jha, A.K., Akhtar, M.J. 2015. Design and application of the CSRR-based planar sensor for noninvasive measurement of complex permittivity. IEEE Sensors Journal, 15: 7181-7189.

Bao, J.Z., Swicord, M.L., Davis, C.C. 1996. Microwave dielectric characterization of binary mixtures of water, methanol, and ethanol. The Journal of Chemical Physics, 104: 4441-4450.

Bia, P., Caratelli, D., Mescia, L., Cicchetti, R., Maione, G., Prudenzano, F. 2015. A novel FDTD formulation based on fractional derivatives for dispersive Havriliak-Negami media. Signal Processing, 107: 312-318.

Chakyar, S.P., Simon, K.S., Bindu, C., Andrews, J., Joseph, V.P. 2017. Complex permittivity measurement using metamaterial split ring resonators. Journal of Applied Physics, 121: 054101.

Chretiennot, T., David, D., Katia, G. 2013. A microwave and microfluidic planar resonator for efficient and accurate complex permittivity characterization of aqueous solutions. IEEE Transactions on Microwave Theory and Techniques, 61: 972-978.

Gupta, S.D., Srivastava, M.C. 2012. Multilayer microstrip antenna quality factor optimization for bandwidth enhancement. Journal of Engineering Science and Technology, 7: 756-773.

Komarov, V., Wang, S., Tang, J. 2005. Permittivity and measurements. Encyclopedia of RF and Microwave Engineering, pp. 3693-3711.
Liu, C., Pu, Y. 2008. A microstrip resonator with slotted ground plane for complex permittivity measurements of liquids. IEEE Microwave and Wireless Components Letters, 18: 257-259.

Luebbers, R., Hunsberger, F.P., Kunz, K.S., Standler, R.B., Schneider, M. 1990. A frequency-dependent finite-difference time-domain formulation for dispersive materials. IEEE Transactions on Electromagnetic Compatibility, 32: 222-227.

Osman, N.A.A., Ibrahim, F., Abas, W.A.B.W., Rahman, H.S.A., Ting, H.N. 2008. $4^{\text {th }}$ Kuala Lumpur International Conference on Biomedical Engineering 2008, vol. 21, pp. 234-241, Springer Science \& Business Media., Kuala Lumpur, Malaysia.

Queffelec, P., Laur, V., Chevalier, A., Le Floch, J.M., Passerieux, D., Cros, D., Houzet, G. 2014. Intercomparison of permittivity measurement techniques for ferroelectric thin layers. Journal of Applied Physics, 115: 024103.

Sarri, A., Batisti, M., Bientinesi, M. 2012. Measurements of complex permittivity of geological materials mixtures at RF frequencies. Antennas and Propagation (EUCAP), $20126^{\text {th }}$ European Conference on. IEEE, 6: 2109-2113.

Sullivan, D.M. 2013. Electromagnetic Simulation using the FDTD Method. John Wiley \& Sons., New Jersey, USA.

Taflove, A., Susan, C.H. 2000. Computational Electrodynamics, Artech House Publishers., Boston, USA.

Yang, C.L., Lee, C.S., Chen, K.W., Chen, K.Z. 2016. Noncontact measurement of complex permittivity and thickness by using planar resonators. IEEE Transactions on Microwave Theory and Techniques, 64: 247-257.

Yee, K. 1966. Numerical solution of initial boundary value problems involving Maxwell's equations in isotropic media. IEEE Transactions on Antennas and Propagation, 14: 302-307.

Yu, K.B., Ogourtsov, S.G., Belenky, V.G., Maslenikov, A.B., Omar, A.S. 2000. Accurate microwave resonant method for complex permittivity measurements of liquids [biological]. IEEE Transactions on Microwave Theory and Techniques, 48: 2159 2164.

Zhadobov, M., Augustine, R., Sauleau, R., Alekseev, S., DiPaola, A., LeQuement, C., Mahamoud, Y.S., LeDrean, Y. 2012. Complex permittivity of representative biological solutions in the $2-67 \mathrm{GHz}$ range. Bioelectromagnetics, 33: 346-355. 